Научная статья

УДК 101.1:316

DOI: $10.18101 / 1994-0866-2021-2-42-47$

\title{
ЦИФРОВИЗАЦИЯ ОБРАЗОВАНИЯ В РОССИИ: РИСКИ И ПРОБЛЕМЫ
}

\author{
(C) Седов Дмитрий Николаевич \\ аспирант, \\ Нижегородский институт управления - филиал Российской академии \\ народного хозяйства и государственной службы \\ при Президенте Российской Федерации \\ Россия, 603950, г. Нижний Новгород, пр. Гагарина, 46 \\ sedoffmail@yandex.ru
}

\begin{abstract}
Аннотация. В данной статье рассмотрены основные риски и проблемы внедрения цифровых инструментов в образовательный процесс. Рассматриваются риски, связанные как с социальной коммуникацией, так и со сферой трудоустройства выпускников вузов, а также проблемы глобализации образовательного процесса. Актуальность исследования подтверждается повышающимся интересом не только научного сообщества к вопросам цифровизации, но и других сфер общественной деятельности. Автор статьи в результате исследования приходит к выводу, что для успешной трансформации образовательной культуры под влиянием процессов цифровизации, подходить ко всем процессам системно и с точки зрения науки, иначе есть высокая вероятность возникновения проблем и понижения эффективности образования в целом. Сделан вывод о том, что процесс цифровизации может как вывести образование из кризисной стадии, так и сделать его неэффективным и усугубить сложившуюся ситуацию.
\end{abstract}

Ключевые слова: цифровизация, цифровизация образования, образовательная культура, диджитализация культуры, цифровое сообщество, цифровое образование

\section{Для цитирования}

Седов Д. Н. Цифровизация образования в России: риски и проблемы // Вестник Бурятского государственного университета. Философия. 2021. Вып. 2. С. 42-47.

Процесс цифровизации стал настоящим трендом XXI в. благодаря своей глобальности и широкому распространению. Цифровизация происходит во всех сферах общества - политике, экономике, культуре, социальной и духовной сфере. В том числе мы наблюдаем резкий рост масштабов применения цифровых технологий в сфере образования, чему сильно способствует мировая пандемия, поставившая учебные заведения в сложные условия дистанционной формы обучения.

Различные аспекты проблемы были исследованы А. Н. Фатенковым [1], который изучал влияние цифровых технологий на жизнь людей в целом и выделял риски потери навыков коммуникации, С. Л. Ивашевским [2], который исследовал трансформацию образовательной культуры и предлагал комплекс мер на уровне государства, А. А. Строковым [3], который оценивал перспективы цифровизации образовательного процесса, и Н. Б. Стрекаловой [5], выявившей основные риски внедрения цифровых технологий в образование. 
Целью проведенного исследования было выявление основных рисков и проблем, которые могли появиться в ходе внедрения цифровых инструментов в образовательный процесс, а также их философский анализ. Для достижения данной цели необходимо было решить две задачи - обозначить основные риски, которые могут повлиять на образовательный процесс, и провести философский анализ трансформации образовательного процесса и его места в жизни человека.

В новой форме обучения - дистанционной - студенты и преподаватели обнаружили немало положительных сторон - экономию времени, возможность использовать специализированное программное обеспечение, подготовить компьютерные проекты и реализовывать учебные идеи онлайн.

Но процесс цифровизации привнес также и отрицательные коррективы, не только учеными-исследователями, но и участниками процесса образования было выявлено много проблем, решение которых остается неочевидным.

Первая проблема - новые образовательные технологии до сих пор не проверены на эффективность. Оценка качества образования участниками образовательного процесса в данный момент низка, в обществе регулярно возникают споры и обсуждения текущей ситуации, и лишь безвыходность положения является фактором, хоть как-то сдерживающим всеобщее негодование.

Эффективность традиционного обучения при соблюдении ряда условий присутствие заинтересованного в результате компетентного педагога, наличие комфортного помещения и оборудования, методологических средств - давно проверена и доказана. Мотивированные и открытые всему новому студенты в таких условиях могут достигнуть своей цели - освоить новую профессию и получить комплексные знания. Принятая еще в Советском Союзе форма обучения позволяла стране подготовить сильные научные кадры и повышать научный потенциал страны [6].

При этом эффективность цифровой формы образования вызывает много вопросов, к процессу обучения глобально не был применен научный подход. Мы отчетливо наблюдаем, как новые технологии «вбрасываются» в процесс обучения вместо планомерного и системного внедрения. Опыт последних лет показывает, что недостаточно просто перенести обучение в онлайн-формат, не изменяя ничего кроме внешней формы. Необходимо также внедрить цифровые инструменты, но какие именно и как - открытый и актуальный вопрос. Помимо этого, необходимо помнить и про цели образования в общем, которые не сводятся только к усвоению новых знаний.

Вторая проблема - обучение с применением цифровых технологий требует доступа к этим технологиям студентов, что потенциально может грозить усложнением доступа к образованию. Очень многие возможности в обществе и так завязаны на финансовом факторе, добавлять к таким возможностям еще и образование будет неверно как минимум с аксиологической точки зрения. Одна из ценностей образования в России - его доступность всем слоям населения ${ }^{1}$, а появ-

${ }^{1}$ Об образовании в Российской Федерации: федеральный закон от 29 декабря 2012 г. № 273-Ф3 с изменениями 2020 г. Текст: непосредственный. 
ление требований к наличию техники определенного уровня потенциально может эту ценность нарушить.

Если цифровизиованной останется лишь часть образовательного процесса, как это происходит во многих университетах сейчас - инновационные технологии применяются выборочно, то вопрос технических ресурсов не является проблемным, но при более глубокой интеграции цифровых технологий в образовательный процесс участникам требуется соблюдение целого ряда технических условий. Специалисты по организации учебного процесса обязательно должны учитывать этот фактор при проектировании образовательного пространства в России.

Конечно, можно подойти к этой проблеме с другой стороны и привести в качестве примера различные проекты открытого образования, например платформу «Открытое образование» ${ }^{1}$. Университеты страны и другие образовательные организации записывают курсы, лекции и другие обучающие материалы, которые в свободном доступе размещаются в интернете, но вопрос доступности таких методов обучения остается открытым — как минимум необходимо соблюсти все технические требования для доступа к курсам.

Третья проблема - вопрос социальной коммуникации становится особенно острым. Цифровые технологии не предполагают очного взаимодействия участников процесса, что позволяет сделать образование дистанционным, но при этом дистанционной становится и сама коммуникация. На первый план в общении и взаимодействии выходит цифровая реальность, занимающая все большее пространство в нашей жизни. При этом нельзя не отметить, что с точки зрения коммуникации она является лишь заместительной формой, а при активизации процессов цифровизации она становится основной и определяющей. Для общества это также является новым вызовом, неизвестно, к каким последствиям это может привести, но риски массовой потери навыков общения и взаимодействия в той или иной степени возможны, а эффективные методы противодействия этим рискам не обозначены до сих пор.

Риск потери навыков коммуникации на данный момент остается высоким даже без учета интеграции цифровых технологий в образовательный процесс. Смартфоны и социальные сети уже во многом вытеснили живое общение, а если учитывать также и широкое применение компьютерных технологий в образовании, то при недостаточно проработанном подходе к данному вопросу мы рискуем получить молодое поколение, практически не способное к эффективной коммуникации, следовательно, к совместной работе, что негативно отразится на всех сферах жизни общества.

Четвертая проблема - с широким распространением цифровых технологий в сфере экономики и бизнеса появляются новые требования к кадрам. От начинающих сотрудников будут требоваться не только базовые теоретические и практические знания по предмету деятельности, но и компетенции по использованию цифровых технологий, а это означает, что требования бизнеса по отношению к высшему образованию будут изменяться. Необходимо будет все больше и больше

\footnotetext{
${ }^{1}$ URL: https://openedu.ru (дата обращения: 21.04.2021). Текст: электронный.
} 
новых компетенций, будущие сотрудники на момент окончания вуза должны обладать большим набором универсальных навыков.

По причине отсутствия опыта, на который образовательная система могла бы опираться, возникает риск несоответствия образовательных программ требуемым цифровым компетенциям у студентов, которые завершили обучение. Цифровизация бизнеса требует внесения значительных изменений в сфере образования, на западе лидеры мировых компаний говорят о слишком большой продолжительности программ высшего образования при их невысокой эффективности.

В решении этой проблемы есть одно сложное препятствие - на данный момент не существует единого портрета современного начинающего специалиста в той или иной сфере, требуемые компетенции по владению цифровыми технологиями не сформулированы однозначно, что затрудняет задачу университетам, которым не на что опираться при формировании новых образовательных программ.

Одним из вариантов решения проблемы может являться более тесное взаимодействие работодателей и образовательных учреждений. Конечно, в образовании есть локальные примеры сотрудничества различных компаний и предприятий с учебными заведениями, но сегодня это не выходит на уровень всей образовательной системы России. В некоторых университетах нашей страны появляются кафедры, открытые предприятиями, например, кафедра корпоративных информационных систем Новосибирского государственного технического университета организована совместно с ООО «IC-Рарус Новосибирск» ${ }^{1}$, но на уровне всего государства таких примеров слишком мало и говорить о новой образовательной тенденции, практикоориентированной, мы полноценно не можем.

Пятая проблема - одной из самых малоисследованных сторон цифровизации является вовлечение в цифровую среду всех участников образовательного процесса, что подлежит детальному проектированию. Процесс образования многогранен и сложен, перенести всю образовательную систему в цифровую среду — масштабная задача, требующая системного подхода.

В настоящее время у нас нет примера университета, который бы успешно и эффективно реализовывал весь процесс обучения онлайн, разработать такой механизм нужно с нуля, а это потенциально содержит множество проблем, рисков и сложностей. Зарубежные вузы уже реализуют некоторые программы дистанционно, опыт реализации отдельных программ в таком формате есть и у российских вузов, но пример полного перехода на данный момент отсутствует.

Да, мы видим успешные примеры университетов, которые реализуют свои программы полностью в онлайн-формате, например Открытый европейский университет в Санкт-Петербурге, но данная организация проводит лишь отдельные курсы, она не выдает дипломов о высшем образовании и не предоставляет услуг по комплексному обучению. Здесь применяется принцип Liberal Arts в чистом виде - студент сам выбирает то, что ему нужно, но все это происходит неофициально, организация никак не подтверждает прохождение обучения, кроме выдачи не имеющего юридической силы сертификата.

${ }^{1}$ URL: https://rarus.ru/nsk (дата обращения: 21.04.2021). Текст: электронный. 
Шестая проблема - одна их самых сложных, соответствие российского образования мировым стандартам. Процессы глобализации, которые в последнее время идут все более активно, влияют и на сферу образования. Университеты по всему миру начинают сотрудничать по большему количеству направлений, создавать совместные проекты, вести совместную научную деятельность. Если российские университеты хотят участвовать в данных процессах, необходимо соответствовать ведущим мировым вузам не только в стандартах оценок или общего качества образования, но и в сфере применения цифровых технологий.

Болонская система до сих пор вызывает множество споров, один из главных аргументов противников системы - выпускники имеют слишком общие знания, не показывают «экспертности» в своих предметах, а если университеты не смогут правильно внедрить цифровые технологии в образовательный процесс, ситуация может ухудшиться, что в конечном итоге может привести к глобальным реформам.

Также многие исследователи отмечают необходимость ввести в России возможность выбора дисциплин студентами, как это наблюдается в Европе и США, осуществить это с применением цифровых технологий вполне возможно, но примеров успешной реализации в нашей стране пока что нет [7].

Подводя итог, отметим, что процесс цифровизации образования уже стал настоящим вызовом для общества. Ввиду постепенного внедрения новых технологий, что необходимо для подготовки высококвалифицированных специалистов, постоянно возникают новые проблемные зоны. И если при планомерном внедрении цифровых технологий многие проблемы еще удается решить, то в 2020 г. в российском образовании произошло экстренное вынужденное внедрение. Вирусная эпидемия внесла свои корректировки в образовательный процесс, который в целях соблюдения мер безопасности сделали дистанционным.

Опросы преподавателей и студентов показывают [8], что образовательная система совершенно не готова к полной цифровой трансформации, форма дистанционного учебного процесса оказалась низкоэффективной, а в некоторых случаях и вовсе не приемлемой при обучении студентов. На первый план вышли проблемы с техникой, квалификацией преподавательского состава, слишком резкое изменение формы образования не позволило грамотно выработать переход с привычной очной формы обучения к дистанционной.

\section{Лuтература}

1. Фатенков А. Н. С цифровыми технологиями - в бесчеловечное будущее // Юридическая наука и практика. Вестник Нижегородской академии MBД России. 2020. № 1(49). URL: https://cyberleninka.ru/article/n/s-tsifrovymi-tehnologiyami-v-beschelovechnoe-buduschee (дата обращения: 08.12.2020). Текст: электронный.

2. Ивашевский С. Л. На пути к изменению образовательных потребностей российского общества // Инновации в образовании. 2007. № 6. С. 22-28. Текст: непосредственный.

3. Строков А. А. Цифровизация образования: проблемы и перспективы // Вестник Мининского университета. 2020. № 2(31). URL: https://cyberleninka.ru/article/ n/tsifrovizatsiyaobrazovaniya-problemy-i-perspektivy (дата обращения: 08.12.2020). Текст: электронный.

4. Ковтуненко Л. В. Актуальные проблемы цифровизации обучения в образовательных организациях ФСИН России // Пенитенциарная наука. 2019. № 2. URL: 
https://cyberleninka.ru/article/n/aktualnye-problemy-tsifrovizatsii-obucheniya-v-obrazovatelnyhorganizatsiyah-fsin-rossii (дата обращения: 08.12.2020). Текст: электронный.

5. Стрекалова Н. Б. Риски внедрения цифровых технологий в образование // Вестник Самарского университета. История, педагогика, филология. 2019. № 2. URL: https://cyberleninka.ru/article/n/riski-vnedreniya-tsifrovyh-tehnologiy-v-obrazovanie (дата обращения: 06.03.2021). Текст: электронный.

6. Шпаковская Л. Л. Советская образовательная политика; социальная инженерия и классовая борьба // Журнал исследований социальной политики. 2009. Т. 7, № 1. С. 39-64. Текст: непосредственный.

7. Боголепова С. В., Малкова Н. В. Liberal Arts в России: непозволительная роскошь или оправданная необходимость // Педагогика. 2017. № 4. С. 80-86. Текст: непосредственный.

8. Общество и пандемия: опыт и уроки борьбы с COVID-19 в России / главный редактор В. А. Мау. Москва, 2020. 744 с. Текст: непосредственный.

Статья поступила в редакциию 29.03.2021; одобрена после рецензирования 29.04.2021; принята к публикации 11.05.2021.

\title{
DIGITALIZATION OF EDUCATION IN RUSSIA: RISKS AND CHALLENGES
}

\author{
Dmitriy N. Sedov \\ Research Assistant, \\ Nizhny Novgorod Institute of Management - \\ branch of Russian Academy of National Economy and Public Administration \\ under the President of the Russian Federation \\ 46 Gagarina Prospect, Nizhny Novgorod 603950, Russia \\ Sedoffmail@yandex.ru
}

Abstract. The article discusses the main risks and problems of introducing digital tools into the educational process of Russia. We have considered the risks associated with the social communication and employment of university graduates, as well as the problems of educational process globalization. The relevance of the study is confirmed by the growing interest of the scientific community in digitalization of science, education, and also other spheres of public activity. As a result of the research, we came to the conclusion that for the successful transformation of educational culture under the influence of digitalization processes it is necessary to approach all processes systematically and from the scientific viewpoint, otherwise there is a high probability of a decrease in the effectiveness of education. It is concluded that digitalization process can both steer the education out of crisis, and make it extremely ineffective.

Keywords: digitalization, digitalization of education, educational culture, digitalization of culture, digital community, digital education

For citation

Sedov D. N. Digitalization of Education in Russia: Risks and Challenges. Bulletin of Buryat State University. Philosophy. 2021; 2: 42-47 (In Russ.).

The article was submitted 29.03.2021; approved after reviewing 29.04.2021; accepted for publication 11.05.2021. 